\title{
Measurement and state preparation via ion trap quantum computing.
}

\author{
S. Schneider, H. M. Wiseman, W. J. Munro, and G. J. Milburn \\ Department of Physics, The University of Queensland, St. Lucia, Brisbane, Qld 4072, Australia
}

\begin{abstract}
We investigate in detail the effects of a QND vibrational number measurement made on single ions in a recently proposed measurement scheme for the vibrational state of a register of ions in a linear rf trap [C. D'Helon and G. J Milburn, Phys. Rev. A 54, 5141 (1996)]. The performance of a measurement shows some interesting patterns which are closely related to searching.
\end{abstract}




\section{INTRODUCTION}

The realisation that quantum physics enables a computer to solve certain problems more efficiently than a classical computer [1] has led to a revision of our understanding of information processing in physical systems. The key feature of quantum physics which lies behind the new developments is, as usual, the superposition principle. In a quantum computer certain properties of a function can be determined, in a single step, by evaluating the function on a coherent superposition of input states. A classical computer in contrast would need to evaluate the function on separate inputs one step at a time. While we are a long way from a complete understanding and classification of quantum algorithms, a number of general features may be distinguished. Firstly, some part of the physical computer, the input register, is used to encode the input states. Typically this involves a set of $N$ two-state systems which may be each prepared in an arbitrary superposition state. Each of these systems is said to encode a qubit, as distinct from the single bit encoded in a classical two state system. As the product Hilbert space of the input system is of dimension $2^{N}$, it may be used to encode a massive amount of information in a single large superposition state. Secondly we identify another part of the physical computer, the output register, to encode the output states. The output register may likewise be a set of two-state systems. Finally there is some unitary transformation which correlates the states of the output register and the input register in such a way that the output register now encodes the result of some function evaluation of the input. The final result is a large entangled state of the input and the output registers. This unitary transformation is a very high dimensional object, and would in general require a very complicated time dependent Hamiltonian. However we now know that an arbitrary unitary transformation may be approximated by a suitable sequence of simpler transformations involving at least two qubits [1].

The scheme described in the previous paragraph has a lot in common with the von Neumann model of a quantum measurement. Instead of the input register, we speak of the measured system, and instead of the output register we speak of the apparatus. However in the von Neumann scheme traditionally, we have only considered unitary transformations generated by a single time independent Hamiltonian. Having seen the quantum computation scheme, this seems to be unnecessarily restrictive. We thus have a novel view of a measurement as an information processing system in which information in the measured system is processed and written to the apparatus. The possibility of choosing a much larger class of unitary transformation so to couple the system and apparatus, and to further process information in the apparatus before read-out, is the motivation of our work.

In a recent paper [2] a method was proposed to make an approximate quantum nondemolition measurement of the number state distribution of the collective vibrational mode of a $N$-qubit ion register. In that paper, a vibrational energy eigenstate, indexed by an integer $n$ (phonon number), was perfectly correlated with the internal states of a number of $N$ trapped ions. A read-out of the internal state of each ion would yield a binary string, of length $N$ encoding a vibrational quantum state and this binary string would occur with the same probability as the vibrational quantum number in the original centre-of-mass state of the ion. In this article we examine what happens if we readout only a subset of the internal states of the trapped ions. We show that when the vibrational mode is prepared in a coherent state, then various measurements on the ions gives various (in general unequally 
weighted) superpositions of coherent states of different phases. In the limit where all ions are measured the ring of superposed coherent states results in a Fock state [3]. We show that this can actually be achieved with a single ion, rather than $N$ ions, if feedback is allowed in the computation. Detailed investigation of the phonon number measurement in terms of filter functions reveals a close relation to the Walsh function generators recently introduced in the context of searching a quantum database [4]. We devote one section to exploring this relation.

\section{THE MODEL}

In this section we summarize the model of D'Helon and Milburn [2]. The physical system consists of $N$ two-level ions forming a 'crystallized' string confined in a linear rf trap. This is our quantum register. We make the usual assumptions that spontaneous emission from the upper internal level of the ions is negligible and that the ions to be cooled into the LambDicke limit. We also neglect decoherence of the center-of-mass motion. The electronic states of the ions are denoted by an integer $k$

$$
|k\rangle_{e}=\left|S_{N}\right\rangle_{N} \otimes\left|S_{N-1}\right\rangle_{N-1} \otimes \ldots \otimes\left|S_{1}\right\rangle_{1}
$$

where $S_{i}=0,1$ and

$$
k=S_{N} \times 2^{N-1}+S_{N-1} \times 2^{N-2}+\ldots+S_{1} \times 2^{0} .
$$

The binary expansion for $k$ is thus just the string $S_{N} S_{N-1} \ldots S_{1}$.

In the model of D'Helon et al. [2], the interaction between the centre-of-mass motion and the internal electronic state of each ion was mediated by a detuned (classical) laser pulse. For interaction times much greater than the vibrational period of the trap, the effective Hamiltonian for the $j$ th ion is

$$
H_{I}^{(j)}=\hbar a^{\dagger} a \chi\left(\sigma_{z}^{(j)}+\frac{1}{2}\right)
$$

where $\sigma_{z}^{(j)}$ is the population inversion for the $j$ th ion and where $\chi=\eta_{C M}^{2} \Omega^{2} /(N \Delta)$. Here $\eta_{C M}$ is the Lamb-Dicke parameter, $\Omega$ is the Rabi frequency for the transitions between the two internal states of the ions, and $\Delta$ the detuning between the exciting laser pulses and the electronic transition. For simplicity we assume that these parameters are the same for all ions.

If we choose the durations $\tau_{j}$ of the standing wave pulses to increase geometrically with qubit number as $\chi \tau_{j}=2^{j} \pi / 2^{N}$, then the Hamiltonians $H_{I}^{(j)}$ generate a unitary transformation

$$
U=\exp \left(\frac{-i 2 \pi a^{\dagger} a \Upsilon}{2^{N}}\right)
$$

where the electronic operator $\Upsilon$ provides a binary ordering of the qubits:

$$
\Upsilon=\sum_{j=1}^{N}\left(\sigma_{z}^{(j)}+\frac{1}{2}\right) 2^{j-1} .
$$


The eigenvectors of the operator $\Upsilon$ are the electronic number states

$$
\Upsilon=\sum_{k=0}^{2^{N}-1} k|k\rangle_{e}\langle k|
$$

In the case of an $N$-ion register, the transformation $U$ does not affect the vibrational state, however it displaces the eigenstates of an electronic operator $\Phi$ which is canonically conjugate to $\Upsilon$. The eigenstates of $\Phi$ are a complementary set of electronic basis states $|\tilde{p}\rangle_{e}$ given by Fourier transforms of the electronic number states $|k\rangle_{e}$,

$$
|\tilde{p}\rangle_{e}=\frac{1}{\sqrt{2}^{N}} \sum_{k=0}^{2^{N}-1} \exp \left(-2 \pi i k p 2^{-N}\right)|k\rangle_{e}
$$

for $0 \leq p \leq 2^{N}-1$

We start with all the ions in the ground state $|0\rangle_{e}$ and an initially arbitrary vibrational state of all the ions, thus

$$
\left|\psi_{i n}\right\rangle=\sum_{n=0}^{\infty} c_{n}|n\rangle_{v i b} \otimes|0\rangle_{e}
$$

We apply a Fourier transform $\mathcal{F}^{-}$to the register, so that it is in the state

$$
\mathcal{F}^{-}|0\rangle_{e}=|\tilde{0}\rangle_{e} \equiv \frac{1}{\sqrt{2^{N}}} \sum_{k=0}^{2^{N}-1}|k\rangle_{e}
$$

which is an equal superposition of all $|k\rangle_{e}$ states and the state of the system now reads

$$
\left|\psi_{i n}^{\prime}\right\rangle=\sum_{n=0}^{\infty} c_{n}|n\rangle_{v i b} \otimes|\tilde{0}\rangle_{e}
$$

As explained in Ref. [2] this can be easily achieved using $\pi / 2$ laser pulses, one for each of the $N$ atoms.

We now apply the unitary transformation in Eq.(蛋 After this interaction we arrive at an entangled state of the vibrational mode and the register

$$
\left|\psi_{\text {out }}^{\prime}\right\rangle=\sum_{n=0}^{\infty} \sum_{p=0}^{2^{N}-1} c_{p+n 2^{N}}\left|p+n 2^{N}\right\rangle_{v i b} \otimes|\tilde{p}\rangle_{e}
$$

where the register is in a superposition of Fourier transformed states. So the final step to get the desired result is now to apply the inverse Fourier transform $\mathcal{F}^{+}$to the register. $\mathcal{F}^{+}$ satisfies $\mathcal{F}^{+} \mathcal{F}^{-}=1$. We get

$$
\left|\psi_{\text {out }}\right\rangle=\mathcal{F}^{+}\left|\psi_{\text {out }}^{\prime}\right\rangle=\sum_{n=0}^{\infty} \sum_{k=0}^{2^{N}-1} c_{k+n 2^{N}}\left|k+n 2^{N}\right\rangle_{\text {vib }} \otimes|k\rangle_{e}
$$

which is the basis for further investigations. 


\section{SUPERPOSITIONS OF COHERENT STATES ON A CIRCLE}

In this section we assume that the vibrational state of the ions is a coherent state

$$
|\psi\rangle_{v i b}=|\alpha\rangle_{v i b}=\sum_{n=0}^{\infty} c_{n}|n\rangle_{v i b}=\sum_{n=0}^{\infty} \frac{\alpha^{k}}{\sqrt{k !}} e^{-\frac{1}{2}|\alpha|^{2}}|n\rangle_{v i b} .
$$

Thus the final state after all the transformations described in section $\mathbb{1}$ is

$$
\left|\psi_{\text {out }}\right\rangle=\sum_{n=0}^{\infty} \sum_{k=0}^{2^{N}-1} \frac{\alpha^{k+n 2^{N}}}{\sqrt{\left(k+n 2^{N}\right) !}} e^{-\frac{1}{2}|\alpha|^{2}}\left|k+n 2^{N}\right\rangle_{\text {vib }} \otimes|k\rangle_{e} .
$$

What happens if we perform a measurement on say the first ion?

We look at the definition of $k$, Eq. 2, and see that the first ion (i.e. ion number one) is determining whether $k$ is even or odd. So measuring ion number one is projecting the register in either a superposition of even or of odd Fock states, depending on the outcome of the measurement. Since the register is highly entangled with the vibrational state of the ions, this measurement will also have an effect on this state: It projects the former coherent state into either an even or odd Schrödinger cat state. This can be seen quite easily by writing the entangled state as a sum of states with even and odd $k$

$$
\begin{aligned}
\left|\psi_{\text {out }}\right\rangle= & \sum_{n=0}^{\infty} \sum_{k=0}^{2^{N-1}-1} \frac{\alpha^{2 k+n 2^{N}}}{\sqrt{\left(2 k+n 2^{N}\right) !}} e^{-\frac{1}{2}|\alpha|^{2}}\left|2 k+n 2^{N}\right\rangle_{v i b} \otimes|2 k\rangle_{e} \\
& +\sum_{n=0}^{\infty} \sum_{k=0}^{2^{N-1}-1} \frac{\alpha^{2 k+1+n 2^{N}}}{\sqrt{\left(2 k+1+n 2^{N}\right) !}} e^{-\frac{1}{2}|\alpha|^{2}}\left|2 k+1+n 2^{N}\right\rangle_{v i b} \otimes|2 k+1\rangle_{e}
\end{aligned}
$$

If we just want to get a Schrödinger cat state, we only need one ion, which means that $N=1$ and the even and odd cats are then entangled with the lower or upper internal level of this ion, respectively. Otherwise we have to disentangle the states again, which can be done by applying $\mathcal{F}^{+} \hat{U}^{-1} \mathcal{F}^{-}$to the measured state.

So what happens if we do not stop after the first ion but go on measuring the second, the third and so on? We just project into more and more superpositions of coherent states aligned around a circle of radius $|\alpha|$. Those superpositions are investigated in [3]. They finally lead to a Fock state in the vibrational motion, depending on how many ions are used. For example, if the last two ions are measured and the result $S_{2}=S_{1}=0$ is obtained, we project the register in a superposition of $|4 k\rangle_{e}$ states and the resulting vibrational state is

$$
\left|\psi_{\text {out }}\right\rangle_{v i b} \propto|\alpha\rangle+|-\alpha\rangle+|i \alpha\rangle+|-i \alpha\rangle
$$

The Wigner function for this is plotted in Fig. 1. Theses results are closely related to work done by Brune et al. [5:6]. There the role of the ions is played by atoms and the phonons by photons in a cavity.

It is interesting to note that the above measurement can in fact be done with a single ion, providing we allow quantum-limited feedback in our computation. Instead of the simultaneous application of the unitary operator (团) on all ions (which requires $N$ different laser pulses on the $N$ ions), one can apply $N$ different laser pulses on the same ion. The scheme 
works as follows: The ion begins in the ground state $|0\rangle$. We apply the Fourier transform operator to the ion, which is simply a $\pi / 2$ pulse:

$$
\mathcal{F}^{-}|0\rangle_{e}=|\tilde{0}\rangle_{e}=(1 / \sqrt{2})\left(|0\rangle_{e}+|1\rangle_{e}\right)
$$

We now transform with the unitary operator

$$
\hat{U}_{j}=\exp \left[-i 2 \pi \hat{a}^{\dagger} \hat{a}\left(\hat{\sigma}_{z}+1 / 2\right) 2^{-N+j-1}\right]
$$

with $j=1$ initially. Then the $-\pi / 2$ pulse is applied, producing the transformation $\mathcal{F}^{+}$. Next, the atom state is read out in the $|0\rangle,|1\rangle$ basis. The result is the least significant digit of $k$ (which determines whether $k$ is even or odd). If the result is 1 we apply the transform $\mathcal{F}^{+}$, if 0 we apply $\mathcal{F}^{-}$. It is these different unitary transformations, controlled by the result of a measurement, which constitute the feedback in the computation. We now move on to the next digit by doubling the interaction time of the pulse in Eq. (18) by setting $j=2$. Following $\mathcal{F}^{-}$we read out the next-to-least significant digit of $k$. The rest follows analogously until we reach $j=N$, where $N$ in this case is completely arbitrary (there is only one ion!).

It might be thought that the measurement would be much quicker with simultaneous pulses on $N$ ions rather than consecutive pulses on a single ion, but this is in fact not the case, as can be seen as follows. Assuming that the $\pi / 2$ pulses can be achieved in negligible time, the time of the measurement will be dominated by the time taken to apply the unitary transformations (4) or (18). As pointed out above, to get the desired unitary transformation the interaction times have to be chosen so that

$$
\chi \tau_{j}=\frac{2^{j} \pi}{2^{N}} .
$$

If the interactions are consecutive (whether on 1 ion or $N$ ions), the total interaction time is

$$
\begin{aligned}
t_{\text {total }} & =\sum_{j=1}^{N} \tau_{j}=\sum_{j=1}^{N} \frac{2^{j} \pi}{2^{N} \chi}=\sum_{j=1}^{N} \frac{2^{j} \pi N \Delta}{2^{N}\left(\eta_{C M} \Omega\right)^{2}} \\
& =\frac{\pi N \Delta}{2^{N}\left(\eta_{C M} \Omega\right)^{2}} 2 \frac{1-2^{N}}{1-2}=\frac{2 \pi N \Delta}{\left(\eta_{C M} \Omega\right)^{2}}\left(1-2^{-N}\right)
\end{aligned}
$$

If the interactions are simultaneous the total interaction time is just the longest interaction time

$$
t_{\text {total }}=\tau_{N}=\frac{\pi N \Delta}{\left(\eta_{C M} \Omega\right)^{2}}
$$

which is at most a fraction of two smaller than the interaction time for consecutive pulses.

From this calculation we can conclude that for making the QND measurement of D'Helon and Milburn one can do perfectly well with one ion, which should make experimental implementation much easier. However it is worth noting that there are some experiments which one could do with the entangled state (12) which are not possible with a single ion. Assume that we do not measure the internal state of the first ion in the register, but perform our 
measurement instead on the second or third or any other ion, and then disentangle the remaining ions by applying $\mathcal{F}^{+} \hat{U}^{-1} \mathcal{F}^{-}$. The result is not as simple as an equally-weighted multiple-component cat-state, but has an interesting interference structure nevertheless. Essentially by measuring one ion (whichever) we set half of the $c_{k+n 2^{N}}$ to zero. For the first ion every second one is set to zero and the result is the standard cat state. For the second ion we get a pattern of $2^{1}$ states set to zero alternating with $2^{1}$ states not set to zero. In general measuring the $i^{\text {th }}$ ion creates a pattern consisting of $2^{i-1}$ states set to zero alternating with the same number of states not set to zero. For example if we measure the second ion to be in the state $|0\rangle$, the register is in a superposition of states of the form $|4 k\rangle_{e}$ and $|4 k+1\rangle_{e}$ $\left(k=0,1,2, \ldots 2^{N-2}-1\right)$ and in this case we project the vibrational state into

$$
\left|\psi_{\text {out }}\right\rangle_{v i b} \propto 2|\alpha\rangle+(1-i)|i \alpha\rangle+(1+i)|-i \alpha\rangle
$$

The Wigner function for this state is plotted in Fig. 2.

\section{SEARCHING FOR A FOCK STATE}

The last considerations in the previous section have a close relation to classical searching as can be seen in [4]. An unsorted database which has one marked entry (i.e. is of Hamming weight one) and contains $2^{N}$ items can be searched classically by bit strings of the same length but Hamming weight $2^{N-1}$. An answer of the database $y$ to the querie $g_{k}$, where $g_{k}$ is the hamming weight $2^{N-1}$ bit string which contains $2^{k}$ zeros alternation with $2^{k}$ ones, is a bit

$$
a_{k}\left(g_{k}, y\right)=g_{k} \cdot y=\left(\sum_{i=1}^{n}\left(g_{k}\right)_{i} y_{i}\right) \bmod 2,
$$

where $\left(g_{k}\right)_{i}$ and $y_{i}$ are the $i^{\text {th }}$ bits of $g_{k}$ and $y$ [4]. Classically the answers of the database to $N$ queries with all the possible Hamming weight $2^{N-1}$ bit strings uniquely determine the location of the marked entry. This location $l$ is just the number we get after transferring the ordered answers (i.e. a binary string of length $N$ ) into their decimal form

$$
l=g_{N-1} \times 2^{N-1}+g_{N-2} \times 2^{N-2}+\ldots+g_{0} \times 2^{0} .
$$

The bit strings $g_{k}$ play an essential rule in the recently introduced algorithm for searching a database [4]. In this work the authors search a database for one marked entry with a single querie which consist of a superposition of Walsh-functions. Walsh-functions are all possible "XOR" combinations of the $g_{k} \mathrm{~s}$ and the bit string containing $n$ zeros. Thus the $g_{k} \mathrm{~s}$ are the generators of the Walsh-functions. Since in [4] the answer to each Walsh-function is stored in the sign of the Walsh-function in the superposition state, further data processing to transform the information into $N$ bits is required.

Now in our scheme we assume that the vibrational motion is in an unknown Fock state $|m\rangle_{v i b}$ and our only knowledge about it is that $m \leq 2^{N}-1$. We choose to implement the measurement using $N$ ions in the trap. The state $(12)$ then reads

$$
\left|\psi_{\text {out }}\right\rangle=\sum_{k=0}^{2^{N}-1} c_{k}|k\rangle_{\text {vib }} \otimes|k\rangle_{e}
$$


where in this special case $c_{k}=\delta_{k, m}$ and so

$$
\begin{aligned}
\left|\psi_{\text {out }}\right\rangle & =|m\rangle_{\text {vib }} \otimes|m\rangle_{e} \\
& =|m\rangle_{\text {vib }} \otimes\left|a_{N-1}\left(g_{N-1}, m\right)\right\rangle_{N}\left|a_{N-2}\left(g_{N-2}, m\right)\right\rangle_{N-1} \ldots\left|a_{0}\left(g_{0}, m\right)\right\rangle_{1} .
\end{aligned}
$$

Measuring the register directly gives the Fock state we were looking for since each ion gives the answer of the database to a querie with a specific generator. This is different from other search schemes [4] where post processing is often required to obtain the information.

How fast can this measurement be done? The preparation of the superposition takes $O(\log (n))$ steps [7]. The interaction presented here also takes a time of order $O(\log (n))$. This is because both equation (20) and equation (21) imply a total time scaling as

$$
t_{\text {total }} \sim N
$$

which is of order $\log (n)$. The inverse Fourier transform however takes $O\left((\log (n))^{2}\right)$ steps [1] which hence is the limiting process. So all in all our measurement scheme takes $O\left((\log (n))^{2}\right)$ steps.

\section{DISCUSSION}

In this paper we have investigated a measurement scheme for the determination of the vibrational quantum number of the lowest normal mode of $N$ ions confined in a trap. The vibrational state of the ions becomes entangled with a state in the product space of the internal electronic states. This product state is defined by an integer encoded as a binary string. The measurement scheme depends on the ability to perform a unitary Fourier transform operation on the product space of the internal electronic states of all the ions. This is a very large dimensional unitary transformation (of dimension $2^{N}$ ), but can be done if we regard the system as an ion trap quantum computer. This enables a significant generalisation of the standard von Neumann measurement model. However we point out that the same measurement can be accomplished with a single ion, provided that subsequent unitary transformations are selected on the basis of past measurements. This is a kind of feedback or adaptive scheme.

The measurement readout is done by reading out the internal electronic state of each ion in some predetermined order. If the excited state encodes a 1 and the ground state encodes a 0 , the result of the measurement is a binary string which encodes an integer $n$. This result will occur with the same probability as the occurrence of the normal mode vibrational quantum number $n$ in the initial centre-of-mass mode. The resulting conditional state of the centre-of-mass is then a Fock state $|n\rangle_{v i b}$. If instead of reading out the entire binary string we only readout a suitable partition by making a state determination on a subset of ions, a number of different vibrational states can be prepared. We have shown for the case of an initial coherent state of the normal mode vibration that the resulting conditional states

are in fact superpositions of coherent states on a ring of fixed radius in the phase-space of vibrational motion.

Determining selected partitions of a binary string is very similar to methods used in binary search routines. We have shown that our measurement scheme can in fact be viewed as a kind of quantum search algorithm for a particular integer $n$ encoding a vibrational normal mode state. 


\section{ACKNOWLEDGMENTS}

S. S. gratefully acknowledges financial support from a "DAAD Doktorandenstipendium

im Rahmen des gemeinsamen Hochschulsonderprogramms III von Bund und Ländern" and from the Center of Laser Science. 


\section{REFERENCES}

[1] A. Ekert and R. Jozsa, Rev. Mod. Physics 68, 733 (1996).

[2] C. D'Helon and G. J. Milburn, Phys. Rev. A 54, 5141 (1996) and C. D'Helon, QuantumLimited Measurements on Trapped Laser-Cooled Ions, PhD thesis, The University of Queensland, 1997.

[3] J. Janszky et al. , Phys. Rev. A 48, 2213 (1993).

[4] B. M. Terhal and J. A. Smolin, submitted to Phys. Rev. A, and Report No. quantph/9705041.

[5] M. Brune et al., Phys. Rev. Lett. 65, 976 (1990).

[6] M. Brune et al., Phys. Rev. A 45, 5193 (1992).

[7] L. K. Grover, Phys. Rev. Lett. 79, 325 (1997). 


\section{FIGURES}

Fig 1: Schneider et. al.

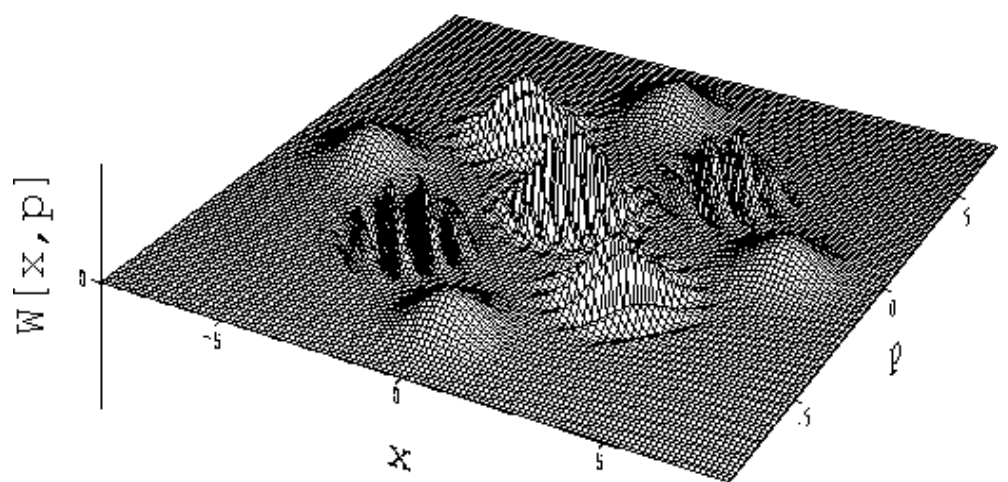

FIG. 1. Wigner function for the state $\left|\psi_{\text {out }}\right\rangle_{v i b} \propto|\alpha\rangle+|-\alpha\rangle+|i \alpha\rangle+|-i \alpha\rangle$ with $\alpha=4.0$.

Fig 2: Schneider et. al.

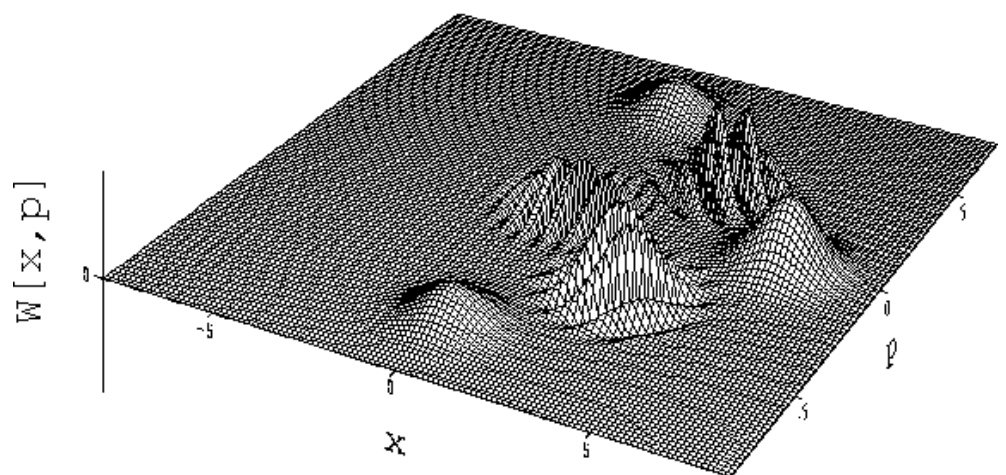

FIG. 2. Wigner function for the state $\left|\psi_{\text {out }}\right\rangle_{v i b} \propto 2|\alpha\rangle+(1-i)|i \alpha\rangle+(1+i)|-i \alpha\rangle$ with $\alpha=4.0$. 